\title{
BMJ Open Help-seeking behaviours among older adults: a scoping review protocol
}

\author{
Kelly Teo (D) , ${ }^{1}$ Ryan Churchill (D) , ${ }^{1}$ Indira Riadi (D) , ${ }^{1}$ Lucy Kervin (D) , \\ Theodore Cosco (D) 1,2
}

To cite: Teo K, Churchill R, Riadi I, et al. Help-seeking behaviours among older adults: a scoping review protocol. BMJ Open

2021;11:e043554. doi:10.1136/ bmjopen-2020-043554

- Prepublication history and additional material for this paper is available online. To view these files, please visit the journal online (http://dx.doi.org/10. 1136/bmjopen-2020-043554).

Received 07 August 2020 Revised 14 January 2021 Accepted 02 February 2021

Check for updates

(c) Author(s) (or their employer(s)) 2021. Re-use permitted under CC BY-NC. No commercial re-use. See rights and permissions. Published by BMJ.

'Department of Gerontology, Simon Fraser University, Vancouver, British Columbia, Canada

${ }^{2}$ Oxford Institute of Population Ageing, University of Oxford, Oxford, UK

Correspondence to

Kelly Teo; kteo@sfu.ca

\begin{abstract}
Introduction Despite evidence that illustrates the unmet healthcare needs of older adults, there is limited research examining their help-seeking behaviour, of which direct intervention can improve patient outcomes. Research in this area conducted with a focus on ethnic minority older adults is also needed, as their help-seeking behaviours may be influenced by various cultural factors. This scoping review aims to explore the global literature on the factors associated with help-seeking behaviours of older adults and how cultural values and backgrounds may impact ethnic minority older adults' help-seeking behaviours in different ways.
\end{abstract}

Methods and analysis The scoping review process will be guided by the methodology framework of Arksey and 0'Malley and the Preferred Reporting Items for Systematic Reviews and Meta-analysis Protocols Extension for Scoping Reviews guidelines. The following electronic databases will be systematically searched from January 2005 onwards: MEDLINE/PubMed, Web of Science, PsycINF0, CINAHL and Scopus. Studies of various designs and methodologies consisting of older adults aged 65 years or older, who are exhibiting help-seeking behaviours for the purpose of remedying a physical or mental health challenge, will be considered for inclusion. Two reviewers will screen full texts and chart data. The results of this scoping review will be summarised quantitatively through numerical counts and qualitatively through a narrative synthesis.

Ethics and dissemination As this is a scoping review of published literature, ethics approval is not required. Results will be disseminated through publication in a peerreviewed journal.

Discussion This scoping review will synthesise the current literature related to the help-seeking behaviours of older adults and ethnic minority older adults. It will identify current gaps in research and potential ways to move forward in developing or implementing strategies that support the various health needs of the diverse older adult population.

Registration This scoping review protocol has been registered with the Open Science Framework (https://osf. io/69kmx).

\section{INTRODUCTION}

Older adults encounter a number of physical and mental health challenges that often require more attention due to their persistent and chronic nature. ${ }^{1}$ As a result, older adults

\section{Strengths and limitations of this study}

To our knowledge, no previous scoping review has explored the help-seeking behaviours of older adults or spoken to the factors that may impact ethnic minority older adults and their help-seeking behaviours differently.

- This study protocol is guided by the methodology framework of Arksey and 0'Malley and will follow a systematic approach to data synthesis.

- The review will be limited to studies published from 2005 onwards to capture both the current health context and the dynamic nature of this topic.

Due to the scoping review design, quality assessments of the included articles will not be performed.

- While there may be little published work in this topic area that will limit the amount of data available, this synthesis of knowledge will serve as a basis for future research and calls to action.

use healthcare services at higher rates and account for more of the healthcare expenditures compared with younger populations. ${ }^{2}$ Despite this, there is concern that not all older adults adequately access healthcare services, and that this population still experiences difficulties in achieving health and have unmet needs. ${ }^{3}$ For example, various studies have found that older adults access mental health services at lower rates than expected, and certain illnesses, such as dementia and glaucoma, continue to go underdiagnosed and undertreated for this group. ${ }^{3-5}$ Reasons for these unmet needs remain unclear in the literature and evidence is scarce. ${ }^{36}$ Woods $e t$ $a \hat{l}$ suggested that unmet care needs among older adults may be attributed to either the limited offers of support from healthcare providers or the lack of acceptance of such support from older adults. Another potential contributor to their unmet needs is that most studies focus on samples of older adults who are already existing service users, thereby limiting the information available on older adults who do not seek help in the first place. ${ }^{6}$ Consequently, there is a clear lack of research examining older adults' decision-making 
processes prior to seeking help as well as what alternatives to formal support services these individuals may use instead. ${ }^{6}$ As such, in exploring unmet needs of older adults, understanding their help-seeking behaviour is one of the direct areas where intervention can improve patient outcomes. ${ }^{7}$

For the purpose of this scoping review, the definition of help-seeking behaviour will be adapted from a WHO study that examined the help-seeking behaviour of adolescents $^{8}$ and will be defined in the following manner ${ }^{9}{ }^{10}$ : any action taken by an older adult who perceives themselves as having a physical or mental health challenge, with the intent to find an appropriate remedy. The type of support that individuals pursue can include seeking formal support services (eg, from clinicians, psychologists, counsellors, religious leaders) or informal support services (eg, from family, friends, the internet).

Within the literature, there are several models that have conceptualised help-seeking behaviour. For example, the health-belief model suggests that an individual's behaviour and desire to change are tied to their beliefs about their health situations and will depend on factors such as their perceived health threat or susceptibility, the benefits of change and the support of internal or external sources (eg, symptoms or mass media). ${ }^{11} 12$ In addition, Andersen's behavioural model of health service utilisation suggests that service use is impacted by several variables; predisposing variables, enabling variables and need variables. ${ }^{12}$ Expansions to this model also recognise how the environment, individual practices and patient satisfaction can influence subsequent help-seeking behaviour. ${ }^{12}$ This scoping review will explore how these various factors may impact the diverse older adult population and discuss which factors may be more salient for this group.

In exploring help-seeking behaviour among older adults, it is important to acknowledge and explore the differing experiences of ethnic minorities, due to their continued lack of representation in clinical and health research. ${ }^{13}$ Ethnicity itself is a topic that has far-reaching cultural effects and encompasses many social issues such as stigma and discrimination that may influence helpseeking attitudes and disparities in care. For example, among ethnic minority adolescents, studies have shown that discrimination by authorities and healthcare providers, traditional and cultural beliefs about mental illness and family obligation values impact their helpseeking behaviour and underutilisation of services. ${ }^{14-16}$ Furthermore, ethnic minority groups have been found to employ alternative and pluralistic help-seeking strategies as opposed to formal primary care services. ${ }^{17}$ Given these factors explored among younger ethnic minority groups, there is reason to believe that attitudes and experiences of help-seeking for ethnic minority older adults are similarly shaped by their cultural backgrounds, values and traditions. Subsequently, these cultural factors influence their health outcomes and the types of support that they prefer to seek out. As such, combined with the challenges that older adults already face with increasing age, there is a need to understand what factors influence ethnic minority older adults' help-seeking behaviour.

This scoping review intends to explore the following research question: Which factors are associated with helpseeking behaviour among older adults? This review also aims to provide answers for the following subquestion: How do cultural backgrounds, values and differences impact help-seeking behaviour among various older adult populations? Due to the exploratory nature of these questions, a scoping review of papers from any country will be conducted to assess the breadth of knowledge in this area. Synthesising this literature can help researchers, healthcare providers and key decision-makers understand the current state of knowledge, identify areas for future research and determine potential ways to influence the help-seeking behaviour of older adults.

\section{METHODS AND ANALYSIS}

This scoping review will be prepared in accordance with the Preferred Reporting Items for Systematic Reviews and Meta-analysis Protocols Extension for Scoping Reviews guidelines. ${ }^{18} \mathrm{~A}$ scoping review is a systematic way of exploring a topic area, in which main concepts and knowledge gaps are identified within a developing field of research. ${ }^{18}$ Unlike systematic reviews, the research questions for a scoping review are often broader and more exploratory $^{18}$ and thus appropriate for this topic due to the limited research and lack of synthesised knowledge.

The review process will be guided by the five main steps set forth by Arksey and O'Malley ${ }^{19}$ and will follow this approximate timeline:

1. Identifying the research question-completed in Fall 2020.

2. Identifying relevant studies-January-February 2021.

3. Study selection-February 2021.

4. Charting the data-March 2021.

5. Collating, summarising, and reporting the resultsApril 2021.

We will aim to complete the optional sixth stage, (6) consultation, by consulting, discussing and verifying the results of this review with older adults. This review has been registered with the Open Science Framework (https://osf.io/69kmx).

\section{Step 1: identifying the research question}

In keeping with the exploratory nature of scoping reviews, we identified one main research question: Which factors are associated with help-seeking behaviour among older adults? As part of this, this review will also explore an additional subquestion: How do cultural backgrounds, values and differences impact help-seeking behaviour among various older adult populations?

\section{Step 2: identifying relevant studies}

The following electronic databases will be systematically searched: MEDLINE/PubMed, Web of Science, PsycINFO, CINAHL and Scopus. Help-seeking behaviour 
is originated from the term 'illness behaviour' in medical sociology. ${ }^{10}$ While one of the earliest definitions of helpseeking behaviour was provided in 1982 and a Willingness to Seek Help Questionnaire was created in 1999, an improved General Help-Seeking Questionnaire was created in 2005 to address previous methodological and instrumental constraints. ${ }^{1020}$ As such, searches will be limited from January 2005 to the date of search commencement to ensure that the literature reflects the current health context and the nature of this dynamic process.

Title, abstract and keyword fields will be searched using a combination of the following terms: ("help seek*" OR "treatment seek*" OR "health information seek*" OR "healthcare seek*" OR "care seek*" OR "health seek*") AND ("older adults" OR "older people" OR "elderly" OR "seniors" OR "geriatrics"). To address the subquestion for ethnic minority older adults, we will also conduct a separate analysis using a combination of the following terms: ("help seek*" OR "treatment seek*" OR "health information seek*" OR "healthcare seek*" OR "care seek*" OR "health seek*") AND ("older adults" OR "older people" OR "elderly" OR "seniors" OR "geriatrics") AND ("immigrants" OR "ethnic minority" OR "minority populations"). An example of the electronic search strategy for MEDLINE/PubMed has been included (online supplemental file 1). The reference lists of the included articles will also be reviewed to ensure that all relevant articles have been included. Additional search strategies may be incorporated as the review progresses and any additions or changes will be documented.

\section{Step 3: study selection}

Two reviewers will screen all articles identified from the databases independently. Titles and abstracts will be reviewed based on the initial search and included based on the eligibility criteria described below. Duplicate articles will be removed; full texts will be examined to create a final list of included studies. Any disagreements on the inclusion of any articles will be resolved through discussion and in consultation with a third reviewer if necessary.

\section{Eligibility Criteria}

Studies of various designs and methodologies (ie, quantitative, qualitative, mixed methods design) will be considered for this review. English or non-English studies from any country must (1) address research on the helpseeking behaviours of older adults, (2) be published from January 2005 to the date of search commencement and (3) be full-text peer-reviewed studies. Articles outside of those defined in the inclusion criteria, such as opinion articles, dissertations, conference proceedings or discussion papers, will be excluded.

In addition, the reviewed literature must include populations of (1) only older adults aged 65 years or older, (2) community-dwelling older adults, (3) older adults experiencing a physical and/or mental health challenge and (4) older adults exhibiting help-seeking behaviours or experiencing barriers/challenges to help seeking. Literature including those aged less than 65 years old, hospitalised patients, older adults living in nursing homes or other long-term care facilities as well as non-human studies will be excluded.

\section{Step 4: charting the data}

Data from the identified studies will be collected and charted according to key themes. From each eligible article, the reviewers will include authorship, year and journal of publication, the general characteristics of participants (ie, age, gender and ethnicity), geographic location of study, study methods, the identified barriers or facilitators associated with help-seeking behaviours, limitations and any other key findings. A combination of Excel and a reference management software will be used to chart the data and to manage the screening process.

\section{Step 5: collating, summarising and reporting the results}

Risk of bias will not be assessed, as this scoping review is only intended to provide an overview of the existing literature, and not to critically appraise the included articles. However, we will include a discussion of any limitations found in the included body of evidence.

The results of this scoping review will be summarised quantitatively through numerical counts (ie, to highlight the amount and type of studies reviewed) and qualitatively through a narrative synthesis. Tables and/or charts will be used to map the study findings and provide an overview, and the screening process will be visualised through a flowchart. Given the exploratory nature of this study, any factors related to help seeking will be considered for review. Facilitators to help seeking will be defined as any factor that has been shown to support or encourage an older adult to seek help for their mental and/or physical health challenges. ${ }^{22}$ Barriers to help seeking will be defined as any factor that has been shown to prevent or discourage an older adult from seeking help for their mental and/or physical health challenges. ${ }^{22}$ These factors could include, but will not be limited to, the following: the presence or absence of caregivers, a lack of resources, stigma, language barriers or cultural values. The discussion will be structured based on the themes that emerge from the review.

\section{Patient and public involvement}

The design of this scoping review protocol did not involve patients or the public, but we will aim to involve older adults in the review process, consulting, discussing and verifying the results of this review.

\section{Ethics and dissemination}

As this scoping review is intended to synthesise the current breadth of knowledge on the help-seeking behaviours of older adults, no ethics approval is required. The results of this scoping review will be disseminated through publication in a peer-reviewed journal. 


\section{DISCUSSION}

The results of this study will establish what is currently known about the help-seeking behaviours of older adults. To our knowledge, this scoping review protocol is the first on this topic and will identify key themes and gaps in understanding how older adults seek support for their physical and mental health needs. The results in turn can serve as the basis for future research, such as a qualitative exploration of older adults' decision-making processes when it comes to seeking formal or informal care, a study on how existing help-seeking facilitators or barriers may be influenced by the current COVID-19 pandemic or a systematic review on specific interventions or strategies that may be effective in improving help-seeking behaviour among various older adult populations. Through better understanding how older adults exhibit help-seeking behaviours and the facilitators or barriers that encourage or discourage them from seeking support, healthcare providers and programme implementers can offer formal healthcare services and programmes that are better tailored to suit the needs of this population. Furthermore, this synthesis of knowledge may also benefit older adult caregivers by highlighting additional supports and formal services that older adults can turn to. In identifying alternative strategies for care, such as home care services or online resources, the burden of caring for older adults does not need to fall solely on their caregivers and loved ones.

A secondary objective of this scoping review is to include the perspectives of ethnic minority older adults and to identify how cultural influences may impact their help-seeking behaviour. Dissecting this information will help policymakers, healthcare decision-makers and programme implementers decide how best to allocate limited healthcare resources, such as providing them with rationale to invest in cultural competency training for staff or offering programmes in multiple languages. This is a significant consideration given the rates of immigration and globalisation worldwide and the importance of ensuring the representation of diverse populations in health research. ${ }^{1323}$ Altogether, this synthesis of literature can support the development of new evidence-based practices that will address the difficulties that older adults may experience when seeking care and provide strategies of support that acknowledge the needs of the incredibly diverse older adult demographic and their varying helpseeking behaviours.

Contributors KT, RC, IR, LK and TC contributed to the development of this manuscript. KT conceptualised the research question, designed the study and prepared the first draft of the manuscript. TC helped refine the research question and provide review expertise. All authors contributed to the refining of the study design, as well as to the editing and revising of this protocol. All authors have approved the final manuscript for submission.

Funding The authors have not declared a specific grant for this research from any funding agency in the public, commercial or not-for-profit sectors.

Competing interests None declared.

Patient consent for publication Not required.
Provenance and peer review Not commissioned; externally peer reviewed.

Supplemental material This content has been supplied by the author(s). It has not been vetted by BMJ Publishing Group Limited (BMJ) and may not have been peer-reviewed. Any opinions or recommendations discussed are solely those of the author(s) and are not endorsed by BMJ. BMJ disclaims all liability and responsibility arising from any reliance placed on the content. Where the content includes any translated material, BMJ does not warrant the accuracy and reliability of the translations (including but not limited to local regulations, clinical guidelines, terminology, drug names and drug dosages), and is not responsible for any error and/or omissions arising from translation and adaptation or otherwise.

Open access This is an open access article distributed in accordance with the Creative Commons Attribution Non Commercial (CC BY-NC 4.0) license, which permits others to distribute, remix, adapt, build upon this work non-commercially, and license their derivative works on different terms, provided the original work is properly cited, appropriate credit is given, any changes made indicated, and the use is non-commercial. See: http://creativecommons.org/licenses/by-nc/4.0/.

\section{ORCID iDs}

Kelly Teo http://orcid.org/0000-0003-2405-6466

Ryan Churchill http://orcid.org/0000-0001-6510-4810

Indira Riadi http://orcid.org/0000-0001-6474-5384

Lucy Kervin http://orcid.org/0000-0002-8711-2173

Theodore Cosco http://orcid.org/0000-0002-9977-8888

\section{REFERENCES}

1 Kovner CT, Mezey M, Harrington C. Who cares for older adults? workforce implications of an aging Society. Health Aff 2002;21:78-89.

2 Zayas CE, He Z, Yuan J, et al. Examining healthcare utilization patterns of elderly middle-aged adults in the United States. Proc Int Fla Al Res Soc Conf 2016;2016:361-6.

3 Woods MD, Kirk MD, Agarwal MS. Vulnerable groups and access to health care: a critical interpretive review. London: National Coordinating Centre NHS Service Delivery Organ RD (NCCSDO), 2005.

4 Lee SJ, Larson EB, Dublin S, et al. A cohort study of healthcare utilization in older adults with undiagnosed dementia. J Gen Intern Med 2018;33:13-15.

5 Mackenzie CS, Scott T, Mather A, et al. Older adults' help-seeking attitudes and treatment beliefs concerning mental health problems. Am J Geriatr Psychiatry 2008;16:1010-9.

6 Canvin K, MacLeod CA, Windle G, et al. Seeking assistance in later life: how do older people evaluate their need for assistance? Age Ageing 2018;47:466-73.

7 Benova L, Grundy E, Ploubidis GB. Socioeconomic position and Health-Seeking behavior for hearing loss among older adults in England. J Gerontol B Psychol Sci Soc Sci 2015;70:443-52.

8 Barker G. Adolescents, social support and help-seeking behaviour: an international literature review and programme consultation with recommendations for action. Geneva: World Health Organization, 2007.

9 Latunji OO, Akinyemi OO. Factors influencing health seeking behaviour among civil servants in Ibadan, Nigeria. Ann Ib Postgrad Med 2018;16:52-60.

10 Rickwood D, Thomas K. Conceptual measurement framework for help-seeking for mental health problems. Psychol Res Behav Manag 2012;5:173-83.

11 Jones CL, Jensen JD, Scherr CL, et al. The health belief model as an explanatory framework in communication research: exploring parallel, serial, and moderated mediation. Health Commun 2015;30:566-76.

12 Aday LA, Andersen RM. Health care utilization and behavior, models of. In: Armitage P, Colton T, eds. Encyclopedia of biostatistics. 2nd ed. Chichester, West Sussex, England: John Wiley \& Sons, Ltd, 2005.

13 George S, Duran N, Norris K. A systematic review of barriers and facilitators to minority research participation among African Americans, Latinos, Asian Americans, and Pacific Islanders. Am J Public Health 2014;104:e16-31.

14 Taylor D, Richards D. Triple jeopardy: complexities of racism, Sexism, and ageism on the experiences of mental health stigma among young Canadian black women of Caribbean descent. Frontiers in Sociology 2019;4.

15 Shefer G, Rose D, Nellums L, et al. 'Our community is the worst': the influence of cultural beliefs on stigma, relationships with family 
and help-seeking in three ethnic communities in London. Int J Soc Psychiatry 2013;59:535-44.

16 Guo S, Nguyen H, Weiss B, et al. Linkages between mental health need and help-seeking behavior among adolescents: moderating role of ethnicity and cultural values. J Couns Psychol 2015;62:682-93.

17 Rüdell K, Bhui K, Priebe S. Do 'alternative' help-seeking strategies affect primary care service use? A survey of help-seeking for mental distress. BMC Public Health 2008;8:207.

18 Tricco AC, Lillie E, Zarin W, et al. PRISMA extension for scoping reviews (PRISMA-ScR): checklist and explanation. Ann Intern Med 2018;169:467-73.

19 Arksey H, O'Malley L. Scoping studies: towards a methodological framework. Int J Soc Res Methodol 2005;8:19-32.
20 Wilson CJ, Deane FP, Ciarrochi JV. Measuring help seeking intentions: properties of the general help seeking questionnaire. Can J Occup Ther 2005;39:15-28.

21 Tuliao AP, Velasquez PA. Revisiting the general help seeking questionnaire: adaptation, exploratory factor analysis, and further validation in a Filipino college student sample. Philipp J Psychol 2014;47:1-17.

22 Gulliver A, Griffiths KM, Christensen H. Barriers and facilitators to mental health help-seeking for young elite athletes: a qualitative study. BMC Psychiatry 2012;12:157.

23 Pakulski J, Markowski S. Globalisation, immigration and multiculturalism - the European and Australian experiences. J Sociol 2014;50:3-9. 